Rev. Elev. Med. Vet. Pays trop., 1966, 19, 4 (567-571).

\title{
Note sur la présence d'aflatoxine dans les fanes d'arachides
}

\author{
par R. BOUdERGUES, H. CALVET, E. DISCACCIATI ef Mme M. CLICHE
}

\begin{abstract}
RÉSUMÉ
Les fanes d'arachide constituent en pays arachidien et spéciolement au Sénégal un aliment appété par les diverses espèces animales. Des recherches effectuées sur ce fourrage au Laboratoire National de l'Elevage et' de Recherches Vétérinaires de Dakar, ont révélé la présence d'aflatoxine de toxicité comparable à celle trouvée dans la graine et les tourteaux d'arachide.
\end{abstract}

Les bovins d'expérience entretenus au Laboratoire national de l'Elevage et de Recherches vétérinaires de Dakar reçoivent traditionnellement comme nourriture de base, un fourrage constitué de fanes d'arachide. Ce même aliment entre dans le rationnement des animaux de laboratoire, lapins, cobayes élevés à la ferme annexe de Sangalkam.

Durant less années 1964-1965, la mortalité survenue sur des zébus et des taurins ndama, à la suite de troubles mal définis, suscite l'intérêt des diverses sections du Laboratoire. Les recherches de bactériologie, virologie, parasitologie, entreprises ne peuvent en élucider la cause.

Durant la même période, l'élevage de cobayes de Sangalkam est décimé par plusieurs enzooties meurtrières dont l'origine demeure également indéterminée.

La répétition de ces accidents ef les lésions anatomo-pathologiques rencontrées font alors envisager l'existence possible d'un facteur toxique dans l'aliment distribué. Etant donné la nature de cet aliment, la fane d'arachide, l'étude s'oriente vers la recherche de l'aflatoxine.

II convient de signaler que STARON et Collab. 1. N. R. A., Path. vég. Versailles (5) ont isolé Aspergillus ochraceus à partir d'un foin stocké dans de mauvaises conditions et ayant provoqué la mort de trois génisses.

Les résultats de ces travaux préliminaires font l'objet de cette note et intéressent les points suivants :
- Extraction et dosage de l'aflatoxine sur :

- un échantillon moyen de paille,

- des échantilions de feuilles,

- des échantillons de tiges,

- des échantillons de coques.

- Contrôle de toxicité des extraits sur :

- caneton,

- cuf embryonné.

- Essais de culture de l'Aspergillus isolé de la paille.

\section{I. - EXTRACTION ET DOSAGE PHYSICO-CHIMIQUE}

Le dosage de l'aflatoxine est effectué selon la méthode préconisée par The Tropical Product Institute (2).

Les fanes d'arachide utilisées sont prélevées sur une livraison arrivant au Laboratoire pour être entreposée dans les greniers. Les fevilles, tiges, racines et coques de la plante Arochis hypogea sont broyées séparément au broyeur Gondard, ainsi qu'un échantillon moyen de paille totale.

$50 \mathrm{~g}$ de broyat sont délipidés pendant $6 \mathrm{~h}$ en soxhlet par de l'éther de pétrole.

Le résidu desséché est traité durant $4 \mathrm{~h}$ par du méthanol, également en soxhlet.

L'extrait méthanolique, concentré à $50 \mathrm{ml}$. est agité avec $25 . \mathrm{ml}$ d'eau et $25 \mathrm{ml}$ de chloror 
forme. La phase chloroformique est décantée et on extrait trois fois la phase méthanol par $25 \mathrm{ml}$ de chloroforme.

Les phases chloroformiques sont réunies ef amenées à un volume de, $100 \mathrm{ml}$.

\section{Chromatographie.}

Lés extraits sont chromatographiés sur Kieselgel $G$ en même temps que des solutions témoins.

\section{Solution témoin $\Pi^{0} 1\left(T_{1}\right)$.}

Aflatoxine $B$ en solution chloroformique, 1 microlitre $=0,0001 \gamma$ d'aflatoxine.

Solution témoin no $2\left(\mathrm{~T}_{2}\right)$.

5 microlitres de $\mathrm{T}_{2}$ contiennent $0,0003 \gamma$ d'aflatoxine $B$ et $0,0003 \gamma$ d'aflatoxine $G$.

La solution $T_{2}$ est la dilution au $1 / 200$ e de la solution obtenue en dissolvant dans $200 \mathrm{ml}$ de chloroforme, $5,6 \mathrm{mg}$ d'un mélange d'aflatoxine $B$ et $G$ (dosé par spectrophotométrie au T.P.I. Londres et contenant 44 p. 100 de $B$ et 44 p. 100 de $G$ ).

\section{Résultats qualitatifs.}

On trouve en lumière $U$. $V$. deux taches fluorescentes :

- une bleu de $\mathrm{Rf}=0,56$,

- une vert jaune de $\mathrm{Rf}=0,40$.

Les pigments végétaux (chlorophylle, etc...) donnent des traînées et des spots rouges en U. V. et jaunes verdâtres én visible. En effectuant une première chromatographie avec du chloroforme, ces traînées peuvent être pratiquement éliminées et amenées à migrer avec le front du solvant.

\section{Résultats quantitatifs.}

\begin{tabular}{|c|c|c|c|c|c|c|}
\hline \multirow{3}{*}{\multicolumn{3}{|c|}{$\begin{array}{ccc}\text { Echantilon moyen de paille } \\
- & - & \text { feuille } \\
- & - & \text { tige } \\
- & - & \text { coque }\end{array} \mid$}} & \multicolumn{4}{|c|}{ Aflatoxines } \\
\hline & & & & B & & $G$ \\
\hline & & & $\begin{array}{l}3 \\
3 \\
0,6 \\
3\end{array}$ & p.p.m. & $\begin{array}{c}6 \\
18 \\
0,9 \\
3\end{array}$ & p.p.m. \\
\hline
\end{tabular}

Notons la forte proportion d'aflatoxine $G$ dans la paille et principalement dans les feuilles.

\section{II. - CONTRÔLE DE TOXICITÉ DES EXTRAITS DE L'ÉCHANTILLON MOYEN}

\section{A. - Sur caneton}

\section{Préparation de l'extrait glucosé.}

L'extrait chloroformique de la paille est mélangé à du glucose ef évaporé à $60^{\circ} \mathrm{C}$ en agitant jusqu'à obtention d'un produit pulvérulent. Ce granulé sec est mis en suspension dans la quantité nécessaire d'eau distıllée pour obtenir par dose :

- Glucose...$\ldots \ldots \ldots \ldots \ldots \ldots .2 \mathrm{~g}$

- Aflatoxine ................ $80 \gamma$

- Equ .................. $5 \mathrm{ml}$

L'extrait chloroformique (P) contient :

- $6 \gamma$ d'aflatoxine B par ml,

- $12 \gamma$ d'aflatoxine $\mathrm{G}$ par $\mathrm{ml}$.

La toxicité de l'aflatoxine $G$ étant 3 fois moindre que celle de $B, 8 \mathrm{ml}$ de l'extrait $P$ ont été utilisés par dose caneton.

La D. L. 50 sur les canetons de 3 jours utilisés au Laboratoire National de l'Elevage à Dakar, se situe en effet aux environs de $80 \mu \mathrm{g} /$ jour (résultat de plusieurs essais de toxicité effectués).

Résultats.

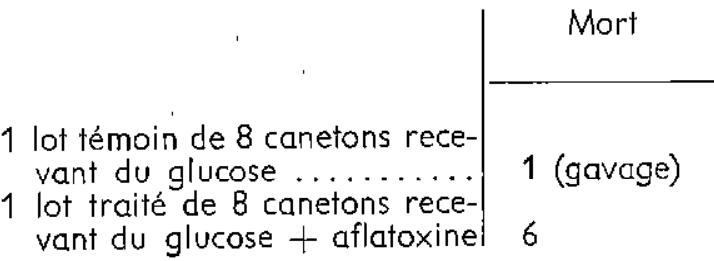

\section{B. - Sur œuf embryonné}

\section{Préparation de l'extrait injecté.}

L'extrait chloroformique, $P$ est passé sur colonne d'alumine basique (*).

Une première élution par du chloroforme élimine la chlorophylle. Une deuxième élution par du méthanol-chloroforme puis du méthanol donne un extrait incolore $P_{1}$ contenant les aflatoxines $B$ et $G$. Des dosages chromatographiques sur plaque Kieselgel $G$ montrent que les afla-

(*) Prolabo. 
toxines $B$ et $G$ se retrouvent dans l'extrait $P_{1}$ qui est dépourvu de chlorophylle.

Les extraits $P_{1}$ sont évaporés et repris par du propylène glycol de façon à obtenir des solutions contenant :

- 0,025 $\gamma$ d'aflatoxine dans $0,04 \mathrm{ml}$.

- 0,050 $\gamma$ d'aflatoxine dans $0,04 \mathrm{ml}$.

Une partie des extraits $P$ subit le même traitement afin de contrôler si la chlorophylle est toxique ou a une action sur la toxicité des aflatoxines.

La technique de VERRET et Coll. (4) a été adoptée. On injecte les extraits à tester dans la poche à air des cufs.

\section{Résultats.}

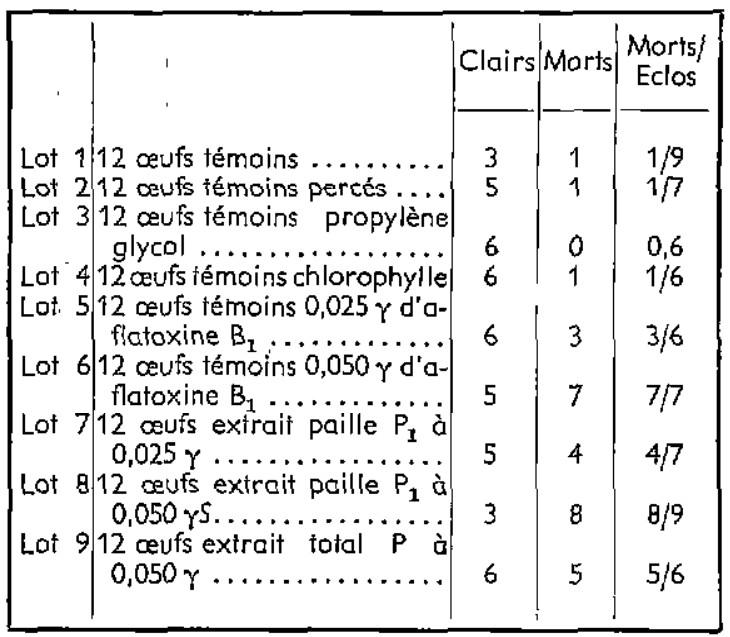

Les extraits paille $P$ et $P_{1}$ ont une toxicıté pour I'œuf embryonné identique aux solutions témoins d'aflatoxine $B_{1}$. La chlorophylle n'a aucune action.

\section{III. - ESSAIS DE CULTURE-ISOLEMENT DE LA SOUCHE}

Des échanfillons de paille sont mis en boîte de Pétri, stérilisés à $110^{\circ} \mathrm{C}$ pendant ving minutes, humidifiés à 20 p. 100 en poids et étuvés à $30^{\circ} \mathrm{C} \pm 1{ }^{\circ} \mathrm{C}$ en atmosphère saturée d'eau pendant 10 jours. II se développe un mycélium, blanchâtre les premiers jours, virant au vert dès le troisième jour; quelques spores sont repiquées sur gélose inclinée de Brian et mises à l'étuve.
A partir de ces souches sur gélose, des arachides broyées sont ensemencées selon la technique décrite dans, le rapport annuel du Laboratoire national de l'Elevage de Dakar, année 1964.

Le dosage physico-chimique des tourteaux d'arachide obtenus, a donné un taux d'aflatoxine $B_{1}$ de 150 p. p. m. et d'aflatoxine $G_{1}$ de 130 p. p. m.

Un deuxième passage sur arachides broyées est effectué à partir de la culture précédente. Le taux d'aflatoxine $B_{1}$ est alors de 250 p. p. m., celui d'aflatoxine $G_{1}$ de 20 p. p. m.

La souche «paille» différente à l'origine s'adapte donc au nouveau milieu de culture constitué par des arachides broyées et les aflatoxines sont retrouvées dans les proportions habituellement obtenues sur ce milieu.

Les essais sur ceuf embryonné de ces extraits d'arachide confirment la toxicité de l'aflatoxine provenant de la souche isolée de la paille d'arachide.

\section{CONTAMINATION DES GRENIERS A FOURRAGE}

Les greniers à fourrage ayant contenu d'importantes quantités de cette paille d'arachide pendant une saison correspondant aux conditions climatiques d'humidité et de température favorables au développement de l'Aspergillus, des essais sont tentés pour mettre en évidence la contamination éventuelle de ces locaux.

Après évacuation de toute la paille d'arachide et un nettoyage sommaire du sol, des échantillons de paille de riz, de foin de prairie de France et d'arachide sont entreposés durant deux mois. Les arachides sont broyées, mises en boîte de Pétri, stérilisées et réhumidifiées ; deux boîtes sont posées sur le sol, deux boîtes témoins mises à l'éfuve.

If faut souligner que les conditions climatiques d'humidité et de température étaient alors moins propices que pendant le stockage de la paille d'arachide.

Ces échantillons repris, stérilisés, humidifiés ef mis en étuve à $30^{\circ} \mathrm{C} \pm 1^{\circ} \mathrm{C}$ en atmosphère saturée d'eau, amènent le développement d'un mycélium vert à partir de l'arachide broyée. Aucune moisissure ne se développe sur les échantillons de paille ainsi que sur les arachides témoins mises à l'étuve. 
L'a contamination des greniers à fourrage paraît donc probable mais' le développement de l'Aspergillus demande un milieu et des conditions de température et d'humidité particulières.

\section{CONCLUSION}

L'aflatoxine, métabolite de Asperglllus flavus, est donc susceptible de se trouver dans les fanes d'arachide, aliment traditionnel du bétail au Sénégal.

Le champignon peut contaminer les locaux de stockage de ces fanes.

Dans le lot examiné, entreposé certainement dans des conditions exceptionnellement mauvaises, le taux du toxique s'avère très élevé. Un bovin consommant couramment de 4 à $5 \mathrm{~kg}$ de ce fourrage absorbe donc 12 à $15 \mathrm{mg}$ d'aflatoxine $B_{1}$. Les tests effectués sur canetons et sur cufs embryonnés montrent que la toxicité de «l'aflatoxine paille» est comparable à celle obtenve sur arachides ensemencées par l'Aspergillus flavus.

Les susceptibilités individuelles mises en évidence dans l'expérimentation sur la vache laitière (1) peuvent expliquer que les accidents survenus sur les bovins, au Laboratoire, aient conservé un caractère sporadique. Mais cette expérimentation semble avoir mis en lumière un autre fait important.

En effet, les taux d' aflatoxine et la nature même de cette aflatoxine sont très différents suivant les diverses parties de la plante. Ce phénomène est difficilement explicable par une contamination survenuie pendant le séchage des fanes, après leur arrachage. On est donc amené à envisager la possibilité d'une invasion de l'arachide par le champignon, durant son cycle végétatif. La concentration du métabolite de l'Aspergillus flavus se faisant dans certaines parties de la plante, dans les fevilles par exemple, 'comme le montrent les analyses.

Cètte hypothèse peut trouver une confirmation dans les résultats analytiques obtenus à la faveur de l'expérimentation agricole sur l'aflatoxine 1964-65 (EXAGRAF I).

En effet, au cours de cette campagne, exécutée sous l'égide de l'UNICEF en vue d'obtenir pour l'alimentation humaine des forines d'arachide exemptes d'aflatoxine, des analyses physicochimiques ont porté sur un certain nombre d'échantillons d'arachide.

Ces échantillons de graine provenaient d'arachides séchées suivant piusieurs procédés. L'innovation essentielle portait sur l'utilisation d'un engin servant à déshydrater l'arachide en totalité, dès arrachage.

Or, sur 46 échantillons provenant de ce dernier mode de séchage, 6 résultats furent positifs à un taux supérieur à 0,05 p. p. m., ce qui démontre la contamination possible de l'arachide avant l'arrachage.

Institut d'Elevage et de Médecine vétérinaire
des Poys tropicaux.
Maisons-Alfort
Laboratoire national de l'Elevage ef de
Recherches vétérinaires,
Dakar-Hann

\section{SUMMARY}

Note on the presence of aflatoxin in groundnut's haulms

In countries where groundnut is grown and specially in Senegal, groundnut's haulm is a good food for various domestic animals.

Researchs made on this fodder at the Laboratoire National de l'Elevage ef de Recherches vétérinaires in Dakar have shown the presence of aflatoxin whose toxicity is comparable to the toxicity evidenced in the groundnut's seed and cake. 


\section{RESUMEN}

Nota sobre la presencia de aflaloxina en las hojarascas de los cacahuetes

En los paises dónde crece el cacahuete, particularmente en Senegal, las hojarascas del cacahuete son un alimento gustado por varias especies animales. Durante las investigaciones efecluadas en el Laboratorio Nacional de la Ganaderia y de las Investigaciones veterinarias de Dakar, se demostró en este pienso la presencia de aflatoxina cuya toxicidad se compara con la encontrada en el grano y en las tortas del cacahueie.

\section{BIBLIOGRAPHIE}

1. CALVET (H.), BOUDERGUES (R.), DISCACCIATI (E.) et Mme CLICHE (M.). - Effets de l'aflatoxine sur les bovins tropicaux. A paraître dans Rev. Elev. Méd. vét. Pays trop.

2. TROPICAL PRODUCTS INSTITUTE.Une méthode de détection de l'aflatoxine dans les arachides et dans les dérivés des arachides (A method for the detection of aflatoxin in groundnuts and groundnuts products). Report no 30/62, 1962, Central Veterinary Laboratory Weybridge, London.

3. SARGEANT (K.), O'KELLY (J.), CARNAGHAN (R. B. A.) and ALLCROFT (R.). Essai du principe toxique de certaines farines d'arachldes (The essay of a toxic principle in certain groundnut meals). Vet. Rec., 1961, 73 (46) : 1219-22.

4. VERRET (J.), MARLIAC (J. P.) and LAUGHIN (J.). - Emploi d'embryon de pouleis pour tester la toxicité de l'aflatoxine (Use of the chickens embryo in the assay of a aflatoxin toxicity). J. A. O. A. C., 1964, 47 (6): 1003. 5. STARON (T.), ALLARD (C.), XUONG (N. D.), CHAMBRE (M. M.), GRABONSKI $(H$.$) and KOLLMANN (A.). - Isolement$ de trols substances à partir de jus de culture ef du mycélium d'Aspergillus ochroceus (Isolation of three toxic substances from culture fluids and from the mycellium of Aspergillus ocheraceus). Phytia. Phytopharm., 1956, 14 (2) : 73-79. 